\title{
DIFFERENCES IN EFFECTS OF PYRROLIZIDINE ALKALOIDS ON FIVE GENERALIST INSECT HERBIVORE SPECIES
}

\author{
MIRKA MACEL, ${ }^{1,3, *}$ MAAIKE BRUINSMA, ${ }^{1,4}$ SANDER M. DIJKSTRA, ${ }^{1}$ \\ TESSA OOIJENDIJK, ${ }^{1}$ HERMANN M. NIEMEYER, ${ }^{2}$ \\ and PETER G. L. KLINKHAMER ${ }^{1}$ \\ ${ }^{1}$ Institute of Biology Leiden, Leiden University, P.O. Box 9516, 2300 RA, Leiden, \\ The Netherlands \\ ${ }^{2}$ Facultad de Ciencias, Universidad de Chile, Casilla 653, Santiago, Chile
}

(Received October 15, 2004; revised February 4, 2005; accepted March 23, 2005)

\begin{abstract}
The evolution of the diversity in plant secondary compounds is often thought to be driven by insect herbivores, although there is little empirical evidence for this assumption. To investigate whether generalist insect herbivores could play a role in the evolution of the diversity of related compounds, we examined if (1) related compounds differ in their effects on generalists, (2) there is a synergistic effect among compounds, and (3) effects of related compounds differed among insect species. The effects of pyrrolizidine alkaloids (PAs) were tested on five generalist insect herbivore species of several genera using artificial diets or neutral substrates to which PAs were added. We found evidence that structurally related PAs differed in their effects to the thrips Frankliniella occidentalis, the aphid Myzus persicae, and the locust Locusta migratoria. The individual PAs had no effect on Spodoptera exigua and Mamestra brassicae caterpillars. For S. exigua, we found indications for synergistic deterrent effects of PAs in PA mixtures. The relative effects of PAs differed between insect species. The PA senkirkine had the strongest effect on the thrips, but had no effect at all on the aphids. Our
\end{abstract}

* To whom correspondence should be addressed. E-mail: mirka.macel@unifr.ch

${ }^{3}$ Current address: Department of Biology, University of Fribourg, Chemin du Musée 10, CH-1700 Fribourg, Switzerland.

${ }^{4}$ Current address: Department of Entomology, Wageningen University, P.O. Box 8031, 6700 EH Wageningen, The Netherlands. 
results show that generalist herbivores could potentially play a role in the evolution and maintenance of the diversity of PAs.

Key Words-Plant-insect interactions, evolution, plant defense, plant secondary metabolites, chemical diversity, Senecio, pyrrolizidine alkaloids, senecionine, senkirkine, deterrent.

\section{INTRODUCTION}

Plants often synthesize a diversity of structurally related secondary metabolites, such as the 34 glucosinolates in Arabidopsis thaliana (Kliebenstein et al., 2001) and the 200 alkaloids in Catharanthus roseus (Mersey and Cutler, 1986). The evolution and maintenance of this diversity in related compounds are poorly understood. Many secondary metabolites act as defense against herbivores, and it is often postulated that insect herbivores play an important role in the evolution of these compounds (Ehrlich and Raven, 1964; Rhoades and Cates, 1976). It has been shown that plant chemistry can indeed be under selection by insect herbivores (Mauricio and Rausher, 1997; Shonle and Bergelson, 2000). One explanation for the diversity of structurally related secondary metabolites is that new compounds evolve in a continuous evolutionary arms race between a plant and its insect herbivores, in which a plant that synthesizes new compounds is able to escape herbivory and insect herbivores, in turn, adapt to these compounds. This coevolutionary model implies that structurally related compounds differ in their effects on herbivores, and that more recently developed compounds in a biosynthetic pathway are more effective against present-day insects (Berenbaum and Feeny, 1981; Miller and Feeny, 1983). If the production of less effective compounds is costly, there would be selection against such compounds, although compounds that are earlier in the biosynthetic pathway can remain in the plant in small quantities as necessary precursors. Additionally, related compounds can act synergistically on herbivores (Adams and Bernays, 1978; Lindroth et al., 1988; Berenbaum et al., 1991). Furthermore, the diversity can be maintained through selection by several different herbivores and/or pathogens (Simms, 1990; Mithen et al., 1995; Juenger and Bergelson, 1998).

Although there are a few examples of differential toxic effects of structurally related compounds on specialist insect herbivores (Berenbaum et al., 1986, 1989; Bowers and Puttick, 1988), other studies have shown that related compounds do not differ in their effects on specialist insects (Lindroth et al., 1988; Johnson et al., 1996, Macel et al., 2002). Agrawal (2000) found that induction of a diversity of glucosinolates in Lepidium virginium had no effect on a specialist but decreased feeding by a generalist herbivore. Less adapted generalist herbivores could be more susceptible to differences in structure among related compounds and, hence, play an important role in the evolu- 
tion and maintenance of the diversity of structurally related secondary plant compounds.

As a model system to study the evolution of the diversity of secondary metabolites, we used the pyrrolizidine alkaloids (PAs) in Senecio species. Senecionine-type PAs, comprising over 100 structures, are abundant in the genus Senecio (Asteraceae) (Hartmann and Witte, 1995), and senecionine is the primary product in the biosynthesis of these structures (Hartmann and Dierich, 1998). Senecio jacobaea L. can contain more than 10 different senecionine-type alkaloids (Witte et al., 1992), and variation in PA patterns has a genetic basis (Vrieling et al., 1993; Macel et al., 2004). PAs are toxic to some mammals (Bull et al., 1968; Mattocks, 1968), have antifungal activity (Hol and van Veen, 2002), and are mutagenic to Drosophila melanogaster (Frei et al., 1992). PAs are known to deter generalist insect herbivores (Bentley et al., 1984; Dreyer et al., 1985; van Dam et al., 1995; Hägele and Rowell-Rahier, 2000). Specialist herbivores can detoxify (Hartmann, 1999; Naumann et al., 2002) and sequester PAs (Rothschild et al., 1979; Dobler, 2001; Pasteels et al., 2003). Larval performance of the specialist moth Tyria jacobaeae L. was not affected by different PA profiles in its host plant S. jacobaea (Macel et al., 2002). Furthermore, larval performance and oviposition preference of this specialist among different Senecio species were not correlated with PA patterns (Macel et al. 2002; Macel and Vrieling, 2003). Host plant choice of PA-sequestering specialist flea beetles of the genus Longitarsus was also not correlated with PA patterns of Senecio species (Kirk et al., unpublished data). These results indicate that it is unlikely that these specialist herbivores play a role in the evolution and maintenance of the diversity of PAs in Senecio species. In this study, we focus on the effects of structurally related PAs on feeding and survival of five generalist insect herbivore species.

We addressed the following questions: (1) do related PAs differ in effects on generalists? (2) Do PAs act synergistically? (3) Do the relative effects of individual PAs (most effective PA) differ between insect species? Survival experiments were performed with the western flower thrips (Frankliniella occidentalis Pergande) and the green pea aphid (Myzus persicae Sulzer) using artificial diets with PAs. The deterrent effects of PAs on insect feeding were tested with the polyphagous Lepidopteran species Spodoptera exigua Hübner and Mamestra brassicae L. and with the gramnivorous migratory locust (Locusta migratoria L.).

\section{METHODS AND MATERIALS}

Pyrrolizidine Alkaloids. Most of the pyrrolizidine alkaloids we used in our experiments belong to the structural group of senecionine-type PAs. To specify 
structural related activity of PAs, we also selected two PAs from other structural groups: heliotrine (lycopsamine-type PA) and monocrotaline (monocrotalinetype PA) (Hartmann and Witte, 1995). Senecionine-type PAs are mostly 12-membered macrocyclic diesters with a retronecine or otonecine base. Lycopsamine-type PAs are monoesters or diesters containing as a necic acid a hydroxylated 2-isopropylbutyric acid, and monocrotaline-type PAs are 11-membered macrocyclic diesters with retronecine base (Hartmann and Witte, 1995). The structures of the pyrrolizidine alkaloids we used are shown in Figure 1. Senecionine, seneciphylline, retrorsine, jacobine, and senkirkine are all present in Senecio species. Senecionine and seneciphylline are commonly found in most Senecio species (Hartmann and Zimmer, 1986; Macel et al., 2002). Jacobine is found in $S$. jacobaea together with senecionine and seneciphylline, and senkirkine is found in $S$. vernalis together with senecionine and seneciphylline. Retrorsine occurs in several Senecio species but is present in large quantities in S. inaequidens. Monocrotaline is found in Crotalaria species and heliotrine in Heliotropum species. Jacobine was isolated by centrifugal partition chromatography (CPC) from a PA extract of flowering plants of $S$. jacobaea extracted as described by Hartmann and Zimmer (1986). Monocrotaline, retrorsine, and retrorsine N-oxide were obtained from Sigma Chemical Co. Senecionine, seneciphylline, and senkirkine were obtained from Roth Chemical Co., and heliotrine was obtained from LATOXAN, France. We tested the effects of

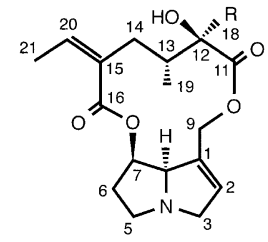

Senecione: $\mathrm{R}=\mathrm{CH}_{3}$ Retrorsine: $\mathrm{R}=\mathrm{CH}_{2} \mathrm{OH}$

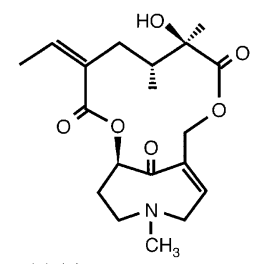

Senkirkine

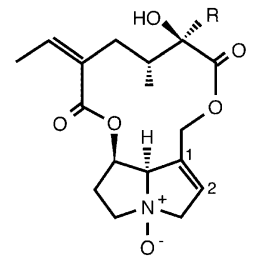

Retrorsine $\mathrm{N}$-oxide: $\mathrm{R}=\mathrm{CH}_{2} \mathrm{OH}$

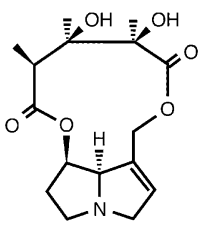

Monocrotaline

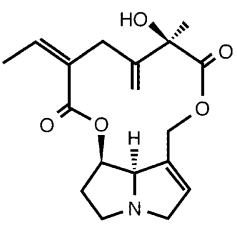

Seneciphylline

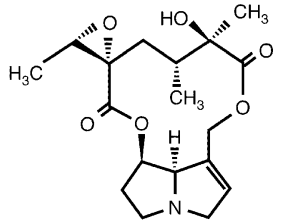

Jacobine

FIG. 1. Structures of pyrrolizidine alkaloids used in this study. Senecionine, $\mathrm{R}=\mathrm{CH}_{3}$, retrorsine: $\mathrm{R}=\mathrm{CH}_{2} \mathrm{OH}$; retrorsine $\mathrm{N}$-oxide, $\mathrm{R}=\mathrm{CH}_{2} \mathrm{OH}$; seneciphylline; jacobine; senkirkine; monocrotaline; heliotrine. 
senecionine, seneciphylline, senkirkine, and monocrotaline on each herbivore. We also tested the other PAs when available. A mixture of senecionine and seneciphylline (commonly found together in Senecio species) was tested for all herbivores but Ma. brassicae. For S. exigua and L. migratoria, other PA mixtures were tested as well. The average concentration of PAs found in $S$. jacobaea plants is $5 \mathrm{mg} \mathrm{g}^{-1}$ dry weight (Vrieling et al., 1993). This concentration was tested for all herbivores. For some herbivores, a range of concentrations of PAs was tested. PA concentrations differ between plant organs. In Senecio vulgaris, PA concentrations range from $0.5 \mathrm{mg} \mathrm{g}^{-1} \mathrm{dw}$ in the leaves to $60 \mathrm{mg} \mathrm{g}^{-1} \mathrm{dw}$ in achenes. In $S$. vernalis, PA concentrations in inflorescences can be $30 \mathrm{mg} \mathrm{g}^{-1}$ dry weight, more than 10 times the concentration found in the leaves (Hartmann and Zimmer, 1986). The effects of tertiary PAs were tested. In plants, PAs mainly occur as N-oxides (Hartmann and Toppel, 1987). After ingestion, the N-oxides are reduced to tertiary PAs in the gut of insects and then passively absorbed (Hartmann, 1999). For retrorsine, we also tested the effects of retrorsine $\mathrm{N}$-oxide.

Frankliniella occidentalis (Thysanoptera; Thripidae). First instars of $F$. occidentalis were obtained from a lab culture reared on chrysanthemum cut flowers in a growth chamber at $20^{\circ} \mathrm{C}, 12$-hr light/dark photoperiod, $60 \% \mathrm{RH}$. For the experiments, we used an artificial diet modified after Singh (1983) by de Jager et al. (1996). PAs were added to the diet by dissolving them in dichloromethane, adding the solution to the diet, and heating the diet $\left(40^{\circ} \mathrm{C}\right)$ to let the dichloromethane evaporate. As a control treatment, we added dichloromethane without PAs to the diet and heated accordingly. For the experiments, we used "observation plates" as described by de Jager et al. (1996). Each plate contained six separate cells, and each cell contained one thrips. On every plate, six larvae could be observed independently. Experiments were done in the same growth chamber in which the thrips were reared. Senecionine, senkirkine, retrorsine, senkirkine, monocrotaline, and heliotrine were used in three different concentrations per PA: $0.5,5$, and $50 \mathrm{mg} \mathrm{g}^{-1} \mathrm{dw}$, equivalent to $0.1 \times, 1 \times$, and $10 \times$ the plant concentration of $S$. jacobaea. Survival of the larvae was measured on 3, 6, 9, and $11 \mathrm{~d}$ after the start of the experiment. Per diet, four observation plates $(N=24)$ were used. In a separate experiment, we used control, retrorsine, and retrorsine $\mathrm{N}$-oxide diets at $10 \times$ plant concentration and measured survival on each diet on $\mathrm{d} 3$ and $\mathrm{d} 6$ of the experiment. Per diet, five observation plates $(N=$ 30) were used. In another experiment, we made diets with a 1:1:1 mixture of senecionine, seneciphylline, and retrorsine, 1:1 mixture of senecionine and seneciphylline, only senecionine, and a control diet. Two concentrations of total alkaloids were used, $2.5 \times$ and $5 \times$ plant concentration. Survival of the thrips larvae was measured on d 3 and $\mathrm{d} 6$ of the experiment. Per diet, five observation plates $(N=30)$ were used. Thrips survival was analyzed with a Cox regression (Fox, 1993). 
Myzus persicae (Homoptera; Aphididae). M. persicae were first reared on chilli pepper plants (Capsicum frutescens). Two months before the experiments, they were transferred to an artificial diet as described by Dadd and Mittler (1966). The aphids were reared in petri dishes (200 aphids per dish) $(5-\mathrm{cm}$ diam), and $350 \mu \mathrm{l}$ of diet were applied between two layers of Parafilm ${ }^{\circledR}$. Once every $3 \mathrm{~d}$, the diet was refreshed. Rearing conditions were $20^{\circ} \mathrm{C}, 12-\mathrm{hr}$ light/ dark photoperiod, $50 \% \mathrm{RH}$. Senecionine, seneciphylline, senkirkine, monocrotaline, and a 1:1 mixture senecionine and seneciphylline were added to artificial diets. To dissolve the PAs in the diets, the $\mathrm{pH}$ was adjusted to $\mathrm{pH} 5$. For each PA, we made four diets of different concentrations: 1.5, 3, 4.5, and 6 $\mathrm{mM}$, equivalent to $0.5 \times, 1 \times, 1.5 \times$, and $2 \times$ the average plant concentration for S. jacobaea. Per experiment, we put 10 adult, apterous aphids on the petri dish. Experiments were repeated 11 times. The experiments lasted $120 \mathrm{hr}$ and were done in a growth chamber at $20^{\circ} \mathrm{C}, 12$-hr light/dark photoperiod, $50 \% \mathrm{RH}$. Not all PAs could be tested simultaneously. We started experiments with three randomly chosen PA diets and a control per day. The mortality on the control diets did not differ significantly among days. Therefore, we grouped the mortality on the control diets on different days together. The percentage mortality of $M$. persicae on the different PA diets was tested against the percentage mortality on control diet with a Mann-Whitney $U$-test with the sequential Bonferroni correction for the number of PA diets we tested.

Spodoptera exigua (Lepidoptera; Noctuidae). Third instar caterpillars from S. exigua were obtained from a lab culture reared on an artificial diet (Singh, 1983 ) in a growth chamber at $25^{\circ} \mathrm{C}, 16: 8$ - hr light/dark photoperiod, $70 \% \mathrm{RH}$. The experiments were performed in the same growth chamber. Senecionine, seneciphylline, retrorsine, retrorsine $\mathrm{N}$-oxide, jacobine, senkirkine, monocrotaline, and heliotrine were dissolved in methanol and applied on lettuce disks (Latuca sativa) of 4-cm diam using a pipette to distribute droplets on the upper side of the lettuce disks. For each PA, three solutions of different concentrations were used: $0.15,0.5$, and $1.5 \mathrm{mg} \mathrm{g}^{-1} \mathrm{fw}$, equivalent to $0.3 \times, 1 \times$, and $3 \times$ the natural PA concentration found in S. jacobaea. As a control, lettuce disks were treated with only methanol. A lettuce disk with a PA was placed on moist filter paper $(4.25-\mathrm{cm}$ diam) in a petri dish $(10-\mathrm{cm}$ diam) and offered to a caterpillar. After $24 \mathrm{hr}$, the amount of lettuce eaten was determined by measuring the leaf area $\left(\mathrm{mm}^{2}\right)$ eaten. The no-choice experiments were repeated 25 times. Differences in amount eaten among the PAs were analyzed with a KruskalWallis test. In a separate experiment, mixtures of two PAs (1:1 senecionine/ seneciphylline, $1: 1$ senecionine/senkirkine), mixtures of three PAs (1:1:1 senecionine/seneciphylline/jacobine, 1:1:1 senecionine, seneciphylline, senkirkine), and the single senecionine were applied to the lettuce disks (total PA concentration $0.5 \mathrm{mg} \mathrm{g}^{-1} \mathrm{fw}$ in all cases). The amount eaten of the disk was normally distributed and analyzed with an ANOVA with post hoc Bonferroni tests. 
Mamestra brassicae (Lepidoptera; Noctuidae). Third and fourth instars from $M a$. brassicae were obtained from a lab culture where they were first reared on white cabbage (Brassica oleracea var. alba). One generation prior to the experiments, they were transferred onto an artificial diet (Singh, 1983) in a growth chamber at $20 / 15^{\circ} \mathrm{C} 16: 8 \mathrm{hr}$ light/dark photoperiod, $70 \% \mathrm{RH}$. Experiments were performed under the same conditions. Senecionine, seneciphylline, retrorsine, retrorsine N-oxide, jacobine, senkirkine, monocrotaline, and heliotrine were dissolved in methanol and applied on lettuce disks of $4.2-\mathrm{cm}$ diam at a concentration of $0.5 \mathrm{mg} \mathrm{g}^{-1} \mathrm{fw}$. The dissolved PAs were applied in the same way as in the Spodoptera experiments. As control, lettuce disks were treated with only methanol. Two lettuce disks, one with a PA and one control, were placed on a moist filter paper $(4.25-\mathrm{cm}$ diam) in a petri dish (14$\mathrm{cm}$ diam) and were offered to a caterpillar in a two-choice experiment. After $24 \mathrm{hr}$, the amount eaten from each lettuce disk was determined by measuring the leaf area $\left(\mathrm{mm}^{2}\right)$ eaten. The choice experiments were repeated 30 times. For each experiment, the preference was tested with the Wilcoxon signedranks matched-pairs test. To be able to compare deterrent effects among PAs, a preference index was generated: preference index $=100 \times$ (amount eaten of PA - amount eaten of control)/(amount eaten PA + amount eaten control).

Differences in preference indices among the PAs were tested with a Kruskal-Wallis test. Post hoc Mann-Whitney $U$-tests were done with a sequential Bonferroni correction for significance levels.

Locusta migratoria (Orthoptera; Acrididae). The fifth instar hoppers of $L$. migratoria (gregarious form) that were used for experiments had been reared on dried grass. The experiments were performed in a growth chamber at 20/ $15^{\circ} \mathrm{C}, 16: 8-\mathrm{hr}$ light/dark photoperiod, $70 \% \mathrm{RH}$. Senecionine, seneciphylline, retrorsine, retrorsine N-oxide, jacobine, senkirkine, monocrotaline, and heliotrine were dissolved in methanol and applied on glass microfiber filters (Whatman ${ }^{\circledR}$ GF/A, 4.7-cm diam). To each filter, $0.5 \mathrm{ml}$ of distilled water was added. The PA concentration was $0.5 \mathrm{mg} \mathrm{g}^{-1} \mathrm{fw}$ ( $\mathrm{fw}$ of filter with water), and to the control, only methanol was added. Two disks, one with PA and one control, were offered in a choice situation to the nymphs. The filters were placed in a white plastic box $(17 \times 12.5 \times 6 \mathrm{~cm})$ covered with a transparent lid. After $16 \mathrm{hr}$, the amount eaten from each filter was determined by measuring the number of $\mathrm{mm}^{2}$ eaten. In the same way, the preference between a single PA and a 1:1(:1) PA mixtures was tested by offering the nymphs a disk with one PA and a disk with a mixture of two or three PAs in a two-choice test. Experiments were repeated 19-26 times. For each experiment, the preference was tested with the Wilcoxon signed-ranks tests. For comparison between PAs, preference indices were calculated and compared in an identical way as for Ma. brassicae. 
F. occidentalis. The survival of the thrips larvae differed among the PA diets. Heliotrine did not affect survival of the larvae, whereas the other PAs significantly reduced larval survival at $10 \times$ plant concentration (Figure 2). At $1 \times$ plant concentration, only senkirkine decreased survival, although this was only marginally significant. Because senecionine is the precursor for senecionine-type PAs, we tested if survival on the senecionine diets differed from the other PAs. Over all concentrations tested, survival on senecionine diets was lower than on the heliotrine diets but higher than on the senkirkine diets (Table 1). We found no indications for a synergistic effect of PAs in PA mixtures. At both $2.5 \times$ and $5 \times$ plant concentration, survival on the PA diets did not differ among diets with only senecionine and diets with PA mixtures (Cox regression, senecionine-mixtures: $P>0.05$ ). Both the retrorsine N-oxide and

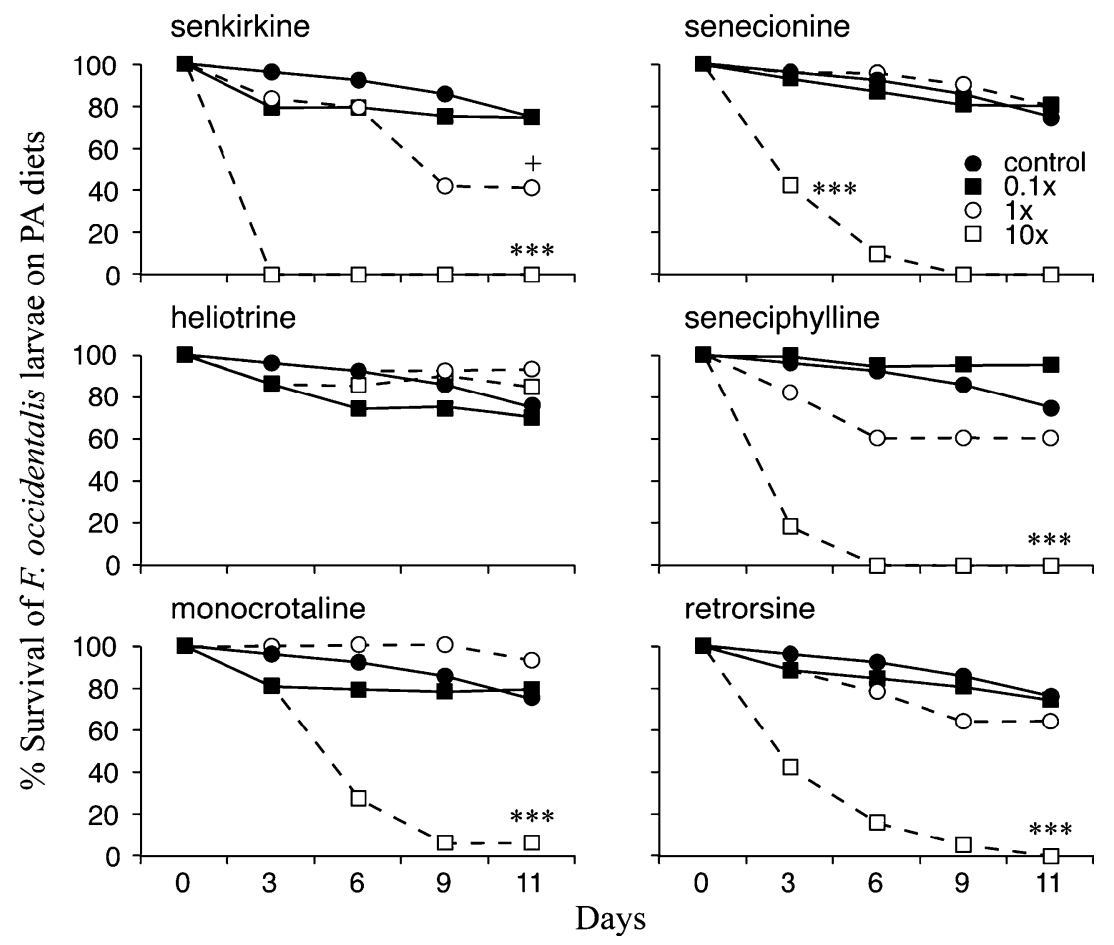

FIG. 2. Percentage survival of larvae of the thrips $F$. occidentalis on diets with pyrrolizidine alkaloids. Three different pyrrolizidine alkaloid concentrations were used, $0.1 \times$, $1 \times$, and $10 \times$ the plant concentration $(N=24$ thrips per diet $) . * * *(P<0.001)^{+}(P<0.07)$ (Cox regression, alkaloid diet compared to control). 
TABle 1. Survival ANAlysis (COX Regression) OF LARVAe of the Thrips F. occidentalis ON PYRROLIZIDINE ALKALOID (PA) DIETS

\begin{tabular}{lrccccc}
\hline & $B$ & SE & Wald & df & P & \multicolumn{1}{c}{ R } \\
\hline Senecionine-PAs & & & 32.70 & 5 & $<0.001$ & 0.129 \\
Retrorsine & 0.192 & 0.265 & 0.525 & 1 & 0.469 & 0 \\
Seneciphylline & 0.199 & 0.280 & 0.504 & 1 & 0.478 & 0 \\
Monocrotaline & -0.355 & 0.289 & 1.508 & 1 & 0.220 & 0 \\
Heliotrine & -1.479 & 0.378 & 15.28 & 1 & $<0.001$ & -0.099 \\
Senkirkine & 0.568 & 0.265 & 4.576 & 1 & 0.032 & 0.043 \\
\hline
\end{tabular}

All alkaloids are tested against senecionine with concentrations as strata in the model. See also Figure 2.

the free-base retrorsine significantly decreased survival compared with the control, but the N-oxide was less toxic than the free base (Figure 3).

$M$. persicae. The survival of $M$. persicae differed among the PA diets (Figure 4). Senecionine and seneciphylline reduced survival at plant concentration levels. Monocrotaline reduced survival only at the highest PA concentration $(2 \times$ plant concentration). Senkirkine did not affect the survival of the aphids at all. There was no synergistic effect of PAs in the PA mixture (Figure 4).

S. exigua. We found no deterrent effects of any individual PA on the feeding of $S$. exigua caterpillars. At all three PA concentrations $(0.3 \times, 1 \times$, and $3 \times$ plant concentration), the amount consumed by the caterpillars from the lettuce disks with PAs did not differ from the control disks or among the PAs (Kruskal-Wallis, all $P>0.30$ ). We did find indications for synergistic effects of PAs in the PA mixtures (Figure 5). The amount eaten tended to differ among the

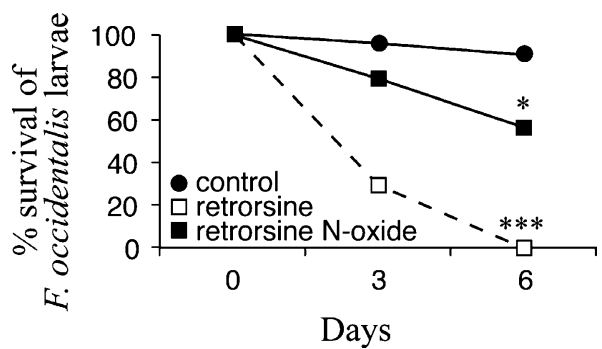

FIG. 3. Percentage survival of larvae of the thrips $F$. occidentalis on diets with retrorsine and retrorsine $N$-oxide and a control diet. PA concentration was $10 \times$ plant concentration $\left(N=30\right.$ thrips per diet). ${ }^{* * *}(P<0.001), *(P<0.05)$ (Cox regression, alkaloid diet compared to control). 


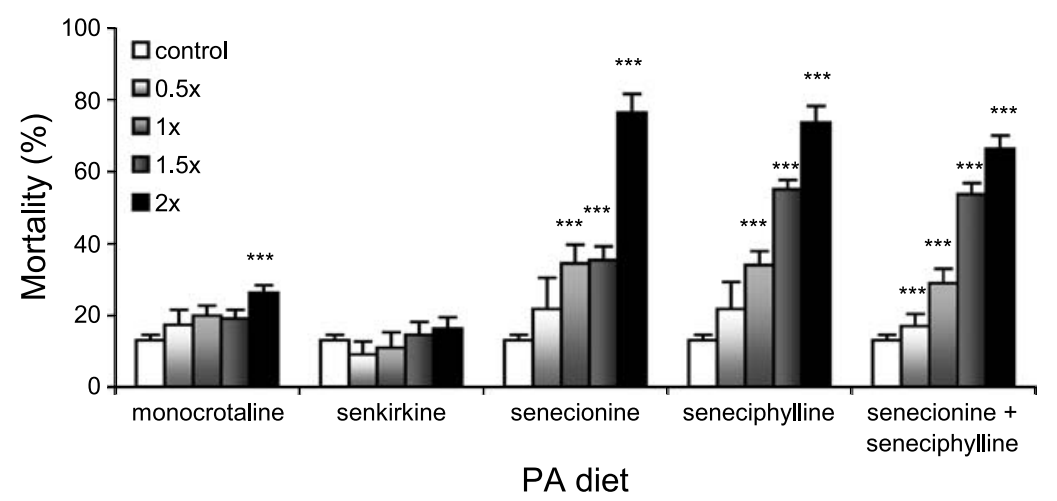

FIG. 4. Survival $( \pm \mathrm{SE})$ of the aphid $M$. persicae on diets with pyrrolizidine alkaloids $(N=$ 11 replicates per diet, 10 aphids per replicate). Concentrations of $0.5 \times, 1 \times, 1.5 \times$, and $2 \times$ the plant concentration of $S$. jacobaea were used. Significance levels of MannWhitney $U$-test for independent samples, alkaloid compared to control, corrected with sequential Bonferroni test: $*(P<0.05),{ }^{* * *}(P<0.001)$.

PA mixtures (ANOVA, $F=2.13, d f=4, P=0.081$ ). Post hoc tests showed that the caterpillars consumed less from the PA mixture of senecionine/seneciphylline/senkirkine than from senecionine. The feeding from the other mixes did not differ significantly from senecionine.

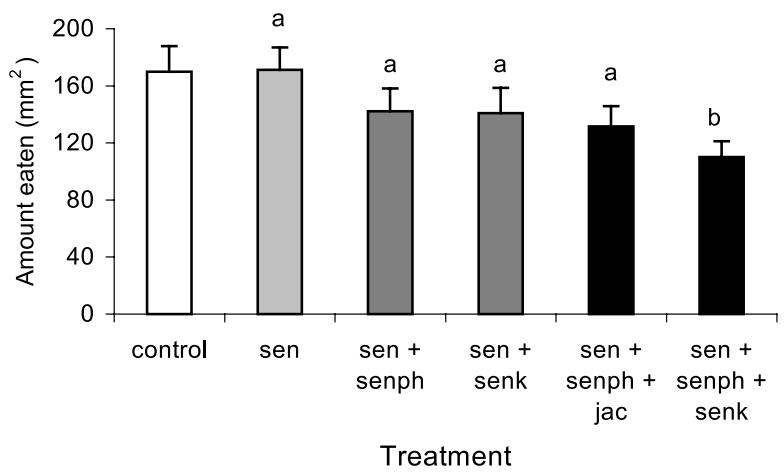

FIG. 5. Amount eaten $( \pm \mathrm{SE})$ by caterpillars of $S$. exigua of lettuce disks with single pyrrolizidine alkaloids, alkaloid mixtures, or control (methanol). Total pyrrolizidine alkaloid concentration was $1 \times$ plant concentration. Different letters indicate significant differences [one-way ANOVA among alkaloids $(P=0.081)$ followed by post hoc Bonferroni tests $(P<0.05)] N=25$ replicates per test. Pyrrolizidine alkaloid codes: sen $=$ senecionine; senph $=$ seneciphylline; senk $=$ senkirkine; jac $=$ jacobine. 


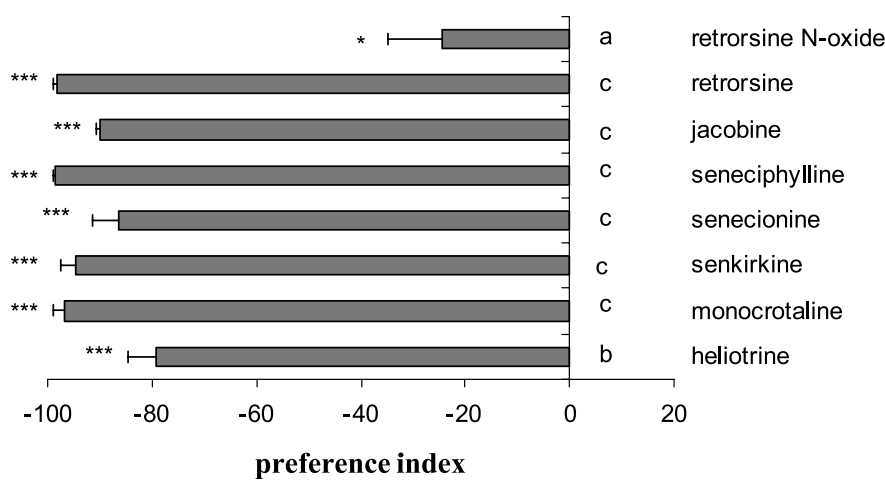

FIG. 6. Preference indices $( \pm \mathrm{SE})$ for L. migratoria feeding on glass fiber filters with pyrrolizidine alkaloids. $N=21-25$ replicates per test. Comparison per alkaloid for testing for preference for control: $*(P<0.05), * * *(P<0.001)$ (Wilcoxon signed-ranks matchedpairs test). Comparison of preference indices: different letters indicate significant differences between preference indices [Mann-Whitney $U$-test with sequential Bonferroni correction $(P<0.05)]$.

Ma. brassicae. No deterrent effects of PAs on the feeding of Ma. brassicae caterpillars were found in any of the two-choice experiments (Wilcoxon signedranks match-pairs tests, all $P>0.05$ ). There were no differences in preference indices between the PAs (Kruskal-Wallis, $\chi^{2}=8.04, d f=6, P=0.24$ ).

L. migratoria. All PAs deterred feeding of L. migratoria, but not all PAs were equally deterrent (Figure 6). Heliotrine was the least deterrent of the freebase PAs. All other PAs were equally deterrent when tested against the control (Figure 6). However, when senecionine and seneciphylline were tested against

TABle 2. FeEding of L. migratoria FROM Glass Fiber DisKS WITH PyRROLIZIDINE ALKALOIDS IN TWO-CHOICE EXPERIMENTS

\begin{tabular}{lccr}
\hline \multicolumn{1}{c}{ Choice } & $\mathrm{mm}^{2}$ eaten $( \pm \mathrm{SE})$ & $N$ & $P^{a}$ \\
\hline Senecionine & $\begin{array}{c}157.9(15.4) \\
10.6(1.2)\end{array}$ & 19 & 0.082 \\
Seneciphylline & $\begin{array}{c}186.0(14.9) \\
4.3(0.3)\end{array}$ & 19 & 0.006 \\
\hline Senecionine & $14.9(11.1)$ & 20 & 0.149 \\
Senecionine + Seneciphylline & $39.9(6.5)$ & & \\
\hline Seneciphylline & $17.3(2.0)$ & 24 & 0.016 \\
Senecionine + Seneciphylline & $2.8(0.3)$ & & \\
\hline Senecionine + Seneciphylline & & & \\
Senecionine + Seneciphylline + Jacobine &
\end{tabular}

${ }^{a} P$ values of Wilcoxon signed-ranks matched-pairs test. 
each other, the locusts tended to prefer senecionine to seneciphylline (Table 2). Senecionine alone was less deterrent than a mixture of senecionine + seneciphylline, but seneciphylline alone was equally deterrent as the senecionine + seneciphylline mixture (Table 2). These results show that seneciphylline is more deterrent than senecionine. The senecionine + seneciphylline mixture was less deterrent than the mixture of senecionine + seneciphylline + jacobine (Table 2), indicating possible synergistic effects. Although the retrorsine $\mathrm{N}$-oxide deterred the locusts, it was less deterrent than the free-base PAs (Figure 6).

\section{DISCUSSION}

Our results showed that the effects of PAs differed among the insect species. All PAs significantly deterred feeding of the locust. Individual PAs did not deter feeding by the caterpillars of the two polyphagous moth species. The two moth species are truly polyphagous and are possibly less sensitive to various secondary metabolites in their diet than the migratory locust that is a specialist on grasses. We performed no-choice tests for $S$. exigua, and choice tests for Ma. brassicae and L. migratoria. Perhaps if we had used choice tests for S. exigua, our results would have been different. van Dam et al. (1995) found that the single PAs from Cynoglossum officinale deterred feeding of $S$. exigua in choice tests. These PAs are of a different structural type than the PAs we tested, which could also explain the contrasting results. The PA N-oxide had less effect on the locust and the thrips than the free-base form of the same PA. Other studies have also shown that PA N-oxides are less deterrent to insects than the free-base PAs (Dreyer et al., 1985; van Dam et al., 1995). Nevertheless, the PA N-oxide still significantly deterred feeding or decreased survival in our experiments. In Senecio species, young leaves and flowers have a relatively high concentration of PAs compared with old leaves (Hartmann and Zimmer, 1986; De Boer, 1999). The concentration-dependent effect of PAs on the aphids and thrips showed that in Senecio species, the more valuable plant parts, flowers, and young leaves are better defended against these generalists than less important organs, as is predicted by the optimal defense theory (Zangerl and Bazzaz, 1992; van Dam et al., 1996).

The coevolutionary arms race hypothesis between plants and insect herbivores (Ehrlich and Raven, 1964) implies that related compounds have different effects on herbivores. Our results showed that structurally related PAs differed in their effects on the locust L. migratoria, the aphid M. persicae, and the thrips $F$. occidentalis, indicating a potential for selection on individual PAs by insects. Differences in (deterrent) effects among structurally related PAs were also found on spruce budworm larvae (Bentley et al., 1984) and the pea aphid Acyrthosiphon pisum (Dreyer et al., 1985). The arms race hypothesis 
further implies that evolutionary older compounds are less effective than more recently developed ones (Feeny, 1976; Berenbaum and Feeny, 1981). It is generally assumed that successive steps in biosynthesis of compounds represent successive evolutionary stages (Rodman, 1981). We found that some, but not all, senecionine-derived PAs were more effective than the precursor senecionine, and this depended on the herbivore species that was tested.

Another explanation for the diversity of plant secondary metabolites could be that related compounds act synergistically (Adams and Bernays, 1978; Lindroth et al., 1988; Berenbaum et al., 1991). However, we did not find any evidence for synergistic effects of PAs on survival of the thrips or aphids. We did find indications that a mixture of PAs is more deterrent than single PAs for the locust and $S$. exigua caterpillars. Therefore, we cannot exclude that plants with a more diverse PA composition will be more successful in deterring generalist insect herbivores than plants with a simpler PA pattern.

The diversity in related secondary metabolites may also be maintained by selection pressure from different herbivores (Simms, 1990; Mithen et al., 1995; Juenger and Bergelson, 1998). The experiments with the thrips and the aphid show that generalist insect species can respond differently to the same PA. While senkirkine was the most effective PA against the thrips at plant concentration level, it had no effect on the aphid. Senecionine and seneciphylline were the most effective against the aphid. Herbivore populations will change over time and, thus, selection pressure on plant defense chemistry will change accordingly. Many authors have linked variation in plant secondary metabolites with contrasting selection pressures from generalist and specialist herbivores (e.g., Linhart, 1991; van der Meijden, 1996). Our results show that it is likely that contrasting selection pressures from different generalist herbivores will also maintain variation in plant secondary compounds. In conclusion, we found that there is a potential for selection of generalist insect herbivores on plant PA profiles and PA diversity.

Acknowledgments - This research was supported by a grant from the Gratama foundation. We thank Helene de Vos for the isolation of jacobine, Lily Muñoz, Henk Nell, and Kees Koops for their technical assistance, Leo Koopman for providing the Mamestra, and Klaas Vrieling, Nicole van Dam, and Ed van der Meijden for their valuable comments on earlier drafts of the manuscript.

\section{REFERENCES}

ADAMS, C. and BERnAYS, E. 1978. The effects of combinations of deterrents on the feeding behavior of Locusta migratoria. Entomol. Exp. Appl. 23:101-109.

Agrawal, A. A. 2000. Benefits and costs of induced plant defense for Lepidium virginicum (Brassicaceae). Ecology 81:1804-1813.

Bentley, M. D., Leonard, D. E., Stoddard, W. F., and Zalkow, L. H. 1984. Pyrrolizidine 
alkaloids as larval feeding deterrents for spruce budworm, Choristoneura fumiferana (Lepidoptera: Tortricidae). Ann. Entomol. Soc. Am. 77:393-397.

BerEnBAuM, M. and FEenY, P. 1981. Toxicity of angular furanocoumarins to swallowtail butterflies: escalation in a coevolutionary arms race? Science 212:927-929.

Berenbaum, M., ZANGerl, A. R., and NitAO, J. K. 1986. Constraints on chemical coevolution: wild parsnips and the parsnip webworm. Evolution 40:1215-1228.

Berenbaum, M., ZANGerL, A. R., and LeE, K. 1989. Chemical barriers to adaptation by a specialist herbivore. Oecologia 18:586-608.

Berenbaum, M., NitAO, J. K., and ZANGerL, A. R. 1991. Adaptive significance of furanocoumarin diversity in Pastinaca sativa (Apiaceae). J. Chem. Ecol. 17:207-215.

Bowers, M. D. and Puttick, G. M. 1988. Response of generalist and specialist insects to qualitative allelochemical variation. J. Chem. Ecol. 14:319-334.

Bull, L. B., Culvenor, C. C. J., and Dick, A. T. 1968. The Pyrrolizidine Alkaloids: Their Chemistry, Pathogenicity and Other Biological Properties. John Wiley \& Sons Inc., New York.

DADD, R. H. and MitTLER, T. E. 1966. Permanent culture of an aphid on a totally synthetic diet. Experientia 22:832-833.

De BoER, N. J. 1999. Pyrrolizidine alkaloid distribution in Senecio jacobaea minimises losses to generalist feeding. Entomol. Exp. Appl. 91:169-173.

De Jager, C. M., BÛtot, R. P. T., VAn Der Meidden, E., and Verpoorte, R. 1996. The role of primary and secondary metabolites in chrysanthemum resistance to Frankliniella occidentalis. J. Chem. Ecol. 22:1987-1999.

DoBler, S. 2001. Evolutionary aspects of defense by recycled plant compounds. Basic Appl. Ecol. 2:15-26.

Dreyer, D. L., Jones, K. C., and MolyneuX, R. J. 1985. Feeding deterrency of some pyrrolizidine, indolizidine, and quinolizidine alkaloids towards pea aphid (Acyrthosiphon pisum) and evidence for phloem transport of indolizidine alkaloid swaisonine. J. Chem. Ecol. 11:1045-1051.

EHrLich, P. R. and RAVEN, P. H. 1964. Butterflies and plants: a study in coevolution. Evolution 18:586-608.

FeEnY, P. 1976. Plant apparency and chemical defense. Recent Adv. Phytochem. 10:1-40.

FoX, G. A. 1993. Failure-time analysis: emergence, flowering, survivorship, and other waiting times, pp. 253-289, in S. M. Scheiner and J. Gurevitch (eds.). Design and Analysis of Ecological Experiments. Chapman and Hall, New York.

Frei, H., LÜthy, J., Brauchli, J., Zweifel, U., WÜrgler, F. E., and Schlatter, C. 1992. Structure/activity relationships of the genotoxic potencies of sixteen pyrrolizidine alkaloids assayed for the induction of somatic mutation and recombination in wing cells of Drosophila melanogaster. Chem. Biol. Interactions 83:1-22.

Hägele, B. F. and Rowell-RAHIER, M. 2000. Choice, performance and heritability of performance of specialist and generalist insect herbivores towards cacalol and seneciphylline, two allelochemicals of Adenostyles alpina (Asteraceae). J. Evol. Biol. 13:131-142.

Hartmann, T. 1999. Chemical ecology of pyrrolizidine alkaloids. Planta 207:483-495.

HARTMANN, T. and DiERICH, B. 1998. Chemical diversity and variation of pyrrolizidine alkaloids of the senecionine type: Biological need or coincidence? Planta 206:443-451.

Hartmann, T. and Toppel, G. 1987. Senecionine N-oxide, the primary product of pyrrolizidine alkaloid biosynthesis in root cultures of Senecio vulgaris. Phytochemistry 26:1639-1643.

HARTMANN, T. and WitTE, L. 1995. Chemistry, biology and chemoecology of the pyrrolizidine alkaloids, pp. 156-233, in S. W. Pelletier (ed.). Alkaloids: Chemical and Biological Perspectives, Vol. 9. Pergamon Press, Elmsford, New York.

HARTMANN, T. and ZiMMER, M. 1986. Organ-specific distribution and accumulation of pyrrolizidine alkaloids during the life history of two annual Senecio species. J. Plant Physiol. 122:67-80. 
Hol, W. H. G. and van VeEn, J. A. 2002. Pyrrolizidine alkaloids from Senecio jacobaea affect fungal growth. J. Chem. Ecol. 28:1751-1760.

JoHNSON, K. S., SCRIBER, J. M., and NAIR, M. 1996. Phenylpropenoid phenolics in sweetbay magnolia as chemical determinants of host use in saturniid silkmoths (Callosamia). J. Chem. Ecol. 22:1955-1969.

Juenger, T. and Bergelson, J. 1998. Pairwise versus diffuse natural selection and the multiple herbivores of scarlet gilia, Ipomopsis aggregata. Evolution 52:1583-1592.

Kliebenstein, D. J., Kroymann, J., Brown, P., Figuth, A., Pedersen, D., Gershenzon, J., and Mitchell-OldS, T. 2001. Genetic control of natural variation in Arabidopsis glucosinolate accumulation. Plant Physiol. 126:811-825.

LiNHART, Y. B. 1991. Disease, parasitism and herbivory: Multidimensional challenges in plant evolution. Trends Ecol. Evol. 6:392-396.

Lindroth, R. L., Scriber, J. M., and HsiA, S. M. T. 1988. Chemical ecology of the tiger swallowtail: mediation of host use by phenolic glycosides. Ecology 69:814-822.

MACEL, M. and VRIELING, K. 2003. Pyrrolizidine alkaloids as oviposition stimulants for the cinnabar moth, Tyria jacobaeae. J. Chem. Ecol. 29:1435-1446.

Macel, M., Klinkhamer, P. G. L., VRieling, K., and VAn Der Meijden, E. 2002. Diversity of pyrrolizidine alkaloids in Senecio species does not affect the specialist herbivore Tyria jacobaeae. Oecologia 133:541-550.

Macel, M., Vrieling, K., and KlinKhamer, P. G. L. 2004. Variation in pyrrolizidine alkaloid patterns of Senecio jacobaea. Phytochemistry 65:865-873.

MATTOCKS, A. R. 1968. Toxicity of pyrrolizidine alkaloids. Nature 217:723-728.

MAURICIO, R. and RAUSHER, M. D. 1997. Experimental manipulation of putative selection agents provides evidence for the role of natural enemies in the evolution of plant defense. Evolution $51: 1435-1444$.

Mersey, B. and Cutler, A. 1986. Differential distribution of specific indole alkaloids in leaves of Catharanthus roseus. Can. J. Bot. 64:1039-1045.

MiLleR, J. S. and FeENY, P. 1983. Effects of benzylisoquinoline alkaloids on the larvae of polyphagous Lepidoptera. Oecologia 58:332-339.

Mithen, R., Raybould, A. F., and Giamoustaris, A. 1995. Divergent selection for secondary metabolites between wild populations of Brassica oleracea and its implications for plant-herbivore interactions. Heredity 75:472-484.

NAUMANN, C., HARTMANN, T., and OBER, D. 2002. Evolutionary recruitment of a flavin-dependent monooxygenase for the detoxification of host plant-acquired pyrrolizidine alkaloids in the alkaloid-defended arctiid moth Tyria jacobaeae. Proc. Natl. Acad. Sci. USA 99:6085-6090.

Pasteels, J. M., Theuring, C., Witte, L., and Hartmann, T. 2003. Sequestration and metabolism of prototoxic pyrrolizidine alkaloids by larvae of the leaf beetle Platyphora boucardi and their transfer via pae into defensive secretions of adults. J. Chem. Ecol. 29:337-355.

RHOADES, D. F. and CATES, R. G. 1976. Toward a general theory of plant antiherbivore chemistry. Recent Adv. Phytochem. 10:168-213.

RoDman, J. E. 1981. Divergence, convergence and parallelism in phytochemical characters: The glucosinolate-myrosinase system, pp. 43-79, in D. A. Young and D. S. Seigler (eds.). Phytochemistry and Angiosperm Phylogeny. Praeger Publishers, New York.

Rothschild, M., Aplin, R. T., Cockrum, P. A., Edgar, J. A., Fairweather, P., and Lees, R. 1979. Pyrrolizidine alkaloids in arctiid moths (Lep.) with a discussion on host plant relationships and the role of these secondary substances in the Arctiidae. Biol. J. Linn. Soc. 12:305-326.

Shonle, I. and Bergelson, J. 2000. Evolutionary ecology of the tropane alkaloids of Datura stramonium L. (Solanaceae). Evolution 54:778-788.

SimMS, E. L. 1990. Examining selection on the multivariate phenotype: Plant resistance to herbivores. Evolution 44:1177-1188. 
SINGH, P. 1983. A general purpose laboratory diet mixture for rearing insects. Insect Sci. Appl. 4:357-362.

VAN DAM, N. M., Vuister, L. W. N., BergshoefF, C., De Vos, H., and VAn Der Meijden, E. 1995. The 'raison d'être' of pyrrolizidine alkaloids in Cynoglossum officinale: Deterrent effects against generalist herbivores. J. Chem. Ecol. 21:507-523.

VAN DAM, N. M., DE JONG, T. J., IwASA, Y., and KUBO, T. 1996. Optimal distribution of defenses: are plants smart investors? Funct. Ecol. 10:128-136.

VAN DER MeIJDEN, E. 1996. Plant defence, an evolutionary dilemma: Contrasting effects of (specialist and generalist) herbivores and natural enemies. Entomol. Exp. Appl. 80:307-310.

VRIELING, K., DE VOS, H., and VAN WiJK, C. A. M. 1993. Genetic analysis of the concentration of pyrrolizidine alkaloids of Senecio jacobaea. Phytochemistry 32:1141-1144.

Witte, L., ERnst, L., Adam, H., and Hartmann, T. 1992. Chemotypes of two pyrrolizidine alkaloid-containing Senecio species. Phytochemistry 31:559-565.

ZANGerl, A. R. and BAZZAZ, F. A. 1992. Theory and pattern in plant defense allocation, pp. 363-391, in R. S. Fritz and E. L. Simms (eds.). Plant Resistance to Herbivores and Pathogens. University of Chicago Press, Chicago. 